\title{
BLASTOCYST DIFFERENTIATION IN VITRO
}

\author{
I. B. WILSON AND E. J. JENKINSON* \\ Department of Zoology, The Brambell Laboratories, \\ University College of North Wales, Bangor, Caernarvonshire
}

Implantation of the blastocyst marks the onset of an intimate association between the mother and embryo which makes the peri-implantation stages particularly inaccessible to investigation. Although extensive studies have now been carried out on the pre-implantation and, to some extent, the postimplantation stages in vitro, little is known of the requirements for growth and differentiation during the implantation period. Such information, and the ability to culture embryos through the implantation stage, would be useful in the evaluation of events at this time.

In the media commonly employed for the culture of preimplantation mouse embryos, which consist of a balanced salt solution (BSS) supplemented with bovine serum albumin (BSA) and energy sources, development normally proceeds to the expanded-blastocyst stage. At this time the blastocyst may hatch from its zona pellucida and remain free-floating in an apparently arrested condition before finally becoming disorganized (Gwatkin, 1966b). In media containing serum, the blastocyst does not remain free-floating and the trophoblast attaches to the floor of the culture vessel producing a flat outgrowth with the cells of the inner cell mass (ICM) remaining on the upper surface (Mintz, 1964; Cole \& Paul, 1965; Gwatkin, 1966a, b). It has been suggested that such outgrowths represent two-dimensional attempts at implantation (Bryson, 1964; Gwatkin, 1966a, b).

The fate of the ICM in these outgrowth cultures is variable. Mintz (1964), using media containing $30 \%$ fetal calf serum (FCS), stated that 'the end-point of in vitro development is a collapse of the blastocyst without endoderm formation and its transformation into a cell culture'. Similarly, Gwatkin (1966a) observed that the development of flattened outgrowths never proceeded to give germ layer formation when embryos were cultured in Eagles medium with 1 to $5 \%$ FCS. Cole \& Paul (1965) found that, when outgrowths were produced in Waymouth's medium supplemented with human and calf serum, the ICM continued to grow as a disorganized mass, its life span being determined by that of the trophoblast (10 to 16 days) on the upper surface of which it was confined. In a small percentage of cases ( 2 to $10 \%$ ), vesicular bodies were formed from the ICM, developing slowly over a period of 10 days. These appeared to have a disorganized internal structure although no detailed examination was made. In contrast to these observations, Barlow \& Sherman (1972) found that by the 6 th day in culture the ICM of outgrowths produced in fortified Eagles medium

* Present address: Department of Pathology, University of Bristol Medical School, University Walk, Bristol BS8 1TD. 
with $10 \%$ FCS had usually disappeared and they concluded that embryonic precursor cells did not fare well under such culture conditions.

In an attempt to overcome the apparent barrier to ICM differentiation which, it was felt, was in part due to the breakdown of organization in the blastocyst, Jenkinson \& Wilson (1970) embedded mouse blastocysts in a transparent three-dimensional protein fibre matrix obtained from the bovine eye lens. In this system initial differentiation to the early egg-cylinder stage was obtained. Recently it has been shown that a similar differentiation of the ICM can take place when blastocysts are implanted on the surface of reconstituted collagen gel in Eagles medium with $10 \%$ FCS (Hsu, 1971). Furthermore, in 5 to $20 \%$ of cases, more extensive differentiation was obtained, with improved embryonic development and the appearance of mesoderm and blood islands in the yolk sac (Hsu, 1971, 1972).

In this paper it is proposed to discuss in more detail the development seen under various conditions in vitro and to examine some of the factors involved in this early differentiation and the reasons for the limitations of the systems in use.

\section{PATTERNS OF DIFFERENTIATION in vitro}

\section{Development in lens explants}

The lens protein of the bovine eye provides a convenient source of jelly-like transparent material in which mouse embryos can be cultured and which provides them with a three-dimensional matrix in which to implant. These cultures can conveniently be carried out by placing a small explant of lens in a deep well slide and injecting blastocysts into it from a glass micropipette. The explant can then be covered with culture medium (Eagles MEM or Earles BSS) supplemented with $10 \% \mathrm{FCS}$ and incubated under $5 \% \mathrm{CO}_{2}$ in air at $37^{\circ} \mathrm{C}$.

The type of development obtained in these cultures after 3 to 5 days is illustrated in Pl. 1, Figs 1 and 2 and Pl. 2, Figs 3 to 6 . In 60 to $80 \%$ of cases the ICM develops to the egg-cylinder stage with the formation of ectoderm, proximal and distal endoderm, and the appearance of the pro-amniotic cavity, although only rarely does this expand. The cells of the trophoblast become vacuolated and giant cell transformation takes place, starting at the abembryonic pole and passing upwards over the blastocyst. In some embryos the trophoblast proliferates over the ICM, indicating the initiation of ectoplacental cone formation. In addition, there are ultrastructural changes in the trophoblast surface, including the loss of microvilli and the appearance of smooth trophoblast membrane. All these changes, which occur independently of maternal influence, are comparable with those seen in vivo.

The termination of development in this system is usually marked by a collapse

\section{EXPLANATION OF PLATE 1}

FIG. 1. L.S. of blastocyst after 3 days culture in bovine lens tissue. I, inner cell mass; P, proximal endoderm; D, distal endoderm; G, trophoblast giant cells.

Fig. 2. Differentiated embryo grown on a collagen surface for 9 days. In the centre the knot of embryonic ectoderm (EE) can be seen with the slit-like pro-amniotic cavity (PA). Above this, the trophoblast has proliferated into the ectoplacental cone (EPC). The outer layer of the vesicle which has collapsed on fixation probably consists of endoderm (P) and represents the expanded yolk sac. 
PLATE 1
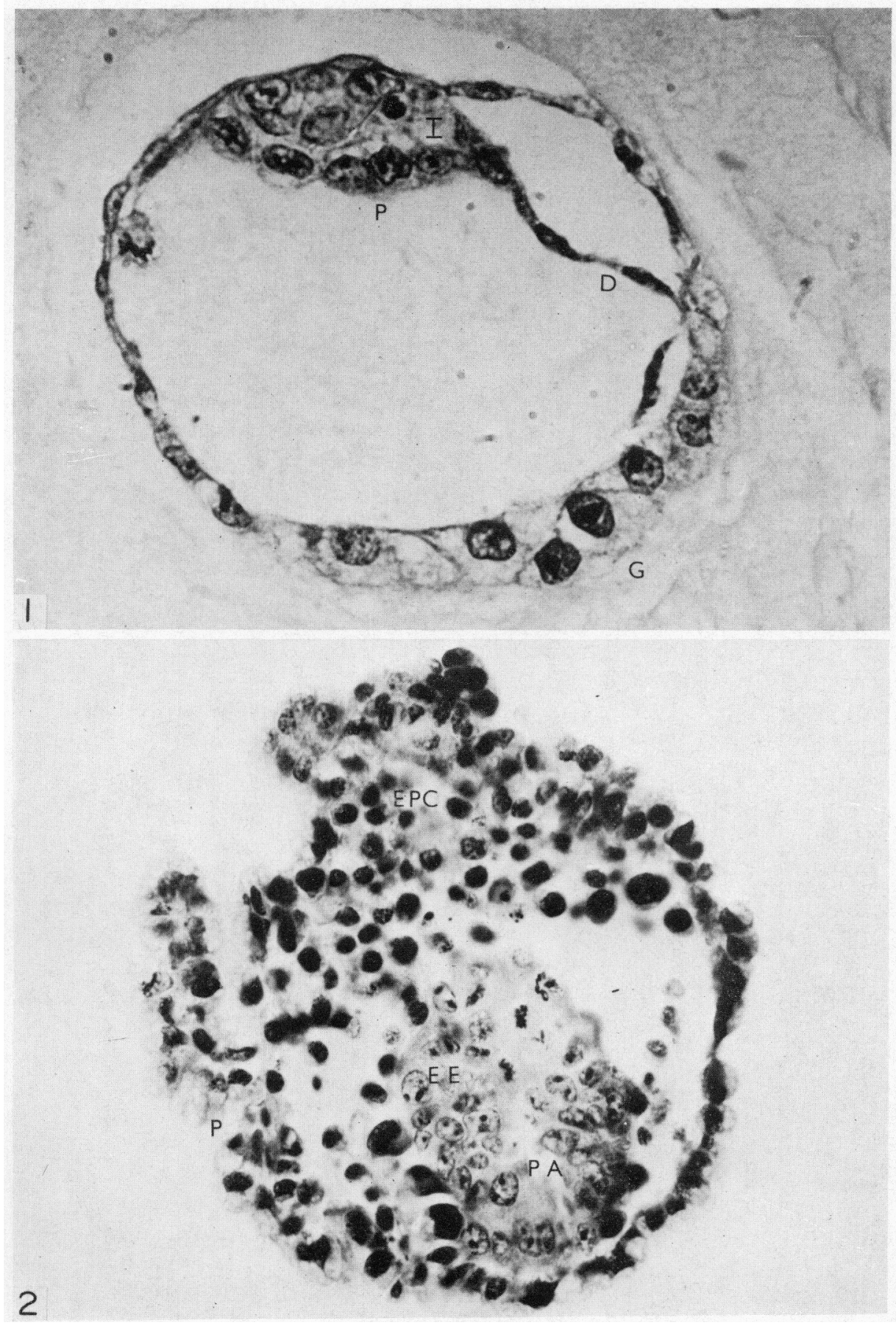

(Facing p. 244) 

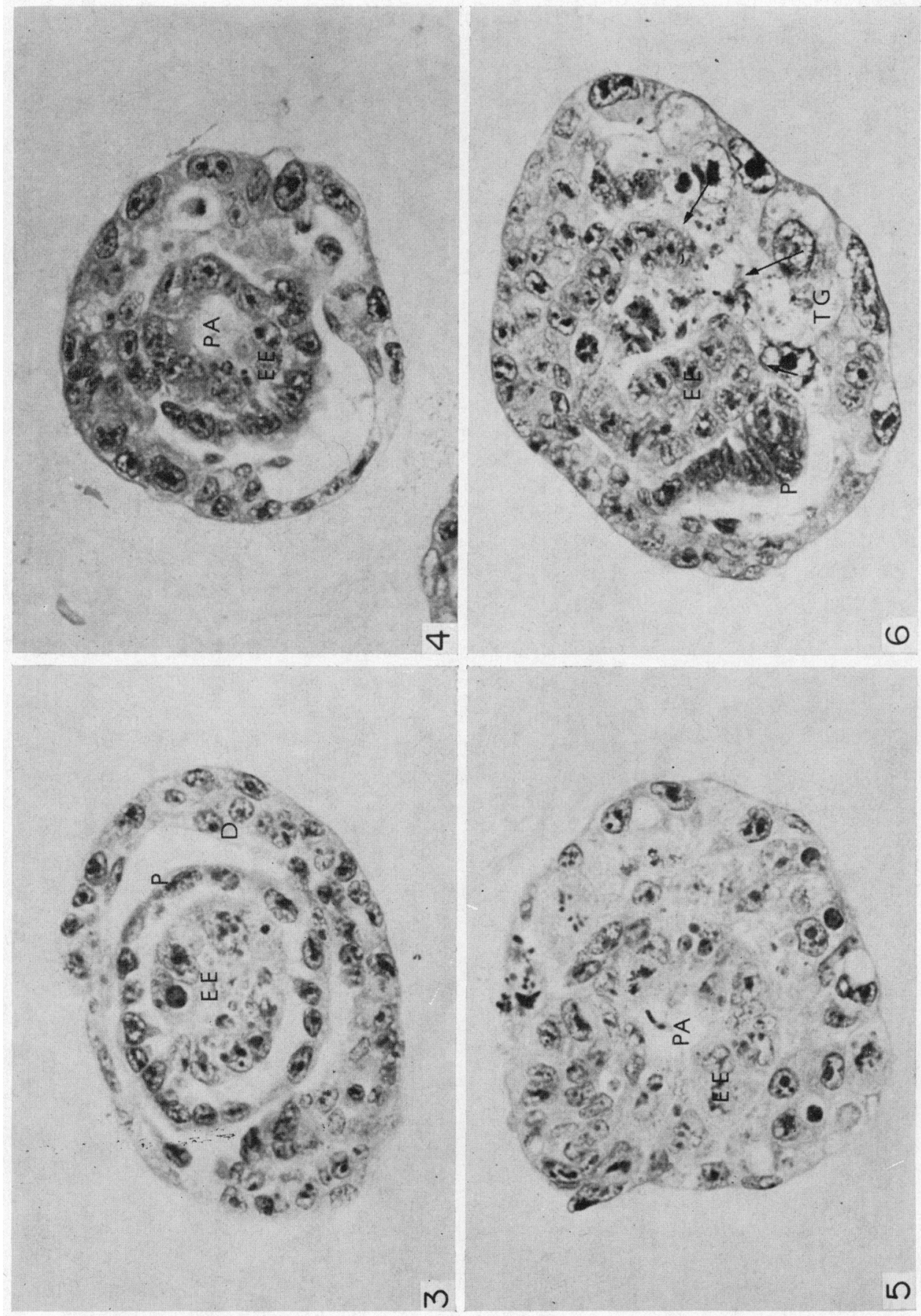
of the blastocyst together with a proliferation of the abembryonic trophoblast which intermingles with the proximal endoderm (Pl. 2, Fig. 5) and may infiltrate the embryonic ectoderm, eventually destroying it (Pl. 2, Fig. 6). The cause of this trophoblastic activity is not clear. If, however, the trophoblast proliferation seen in ectoplacental cone formation is due to the inductive influence of the underlying ICM (Gardner, 1971), then collapse of the blastocyst would bring all the trophoblast in contact with the ICM and induce proliferation all around the circumference. It also seems reasonable to propose that the invasion of embryonic ectoderm by the trophoblast results from the absence of Reichert's membrane, which does not develop in these cultures. This would suggest that an important function of this structure, which normally forms an acellular envelope enclosing the embryo, is to isolate the ICM from the trophoblast at the time this tissue acquires its invasive properties.

\section{Development on collagen surfaces}

Reconstituted rat-tail collagen prepared either by the method of Ehrmann \& Gey (1956) or that of Elsdale \& Bard (1972) and used as a coating on plastic Petri dishes also provides a substrate on which blastocysts can implant. In our experience the great majority of embryos cultured on collagen surfaces in serum-supplemented medium behave in a similar manner to that described by most previous workers. The trophoblast produces its characteristic outgrowth while the ICM remains on the upper surface as a disorganized group of non-differentiated cells showing some proliferation. Where several blastocysts are cultured in close proximity the trophoblastic outgrowths can become confluent and the ICM cells on the upper surface fuse into a large mass which may develop into a vesicular yolk sac-like structure. This ability of the ICMs to fuse emphasizes the lability of these cells at a time when the trophoblast is already capable of implantation (see Gardner, 1971).

In a small percentage of cases (about $10 \%$ ), we have obtained development on collagen surfaces similar to that described by Hsu (1971), although mesoderm formation did not occur (Pl. 1, Fig. 2). Recently, Hsu (1972), using large numbers of embryos, has obtained more advanced differentiation on collagen surfaces, including embryonic development up to the somite stages. The degree and incidence of this development, however, appear to be variable and sporadic, occurring on average in 15 to $20 \%$ of cases. The reason for the sporadic nature of this development is unknown. Hsu considers that the critical stage in these cultures is the differentiation of the ICM into endoderm and ectoderm, only those embryos achieving this differentiation being capable of further

\section{EXPLANATION OF PLATE 2}

Fig. 3. T.S. of egg cylinder after 4 days in lens culture. EE, embryonic ectoderm; $P$, proximal endoderm; $D$, distal endoderm.

FIG. 4. Similar embryo to that shown in Fig. 3 showing the appearance of the proamniotic cavity (PA) in the centre of the embryonic ectoderm (EE).

Frg. 5. T.S. of egg cylinder after 5 days in lens culture. The trophoblast has mingled with the proximal endoderm although the embryonic ectoderm (EE) surrounding the pro-amniotic cavity (PA) is still discrete.

FIG. 6. Oblique section of egg cylinder after $4 \frac{1}{2}$ days in lens culture. The embryonic knot consisting of ectoderm (EE) and endoderm (P) can still be seen but trophoblast giant cells (TG) are pushing inwards into the embryo (arrows). 
development. On the other hand, we have found that most embryos can develop to this stage in lens cultures and that the critical step is expansion of the proamniotic cavity. While nutrient factors play a rôle in successful development (see below), physical factors affecting the maintenance of organization in the embryo are probably also important. In this respect a comparison of the lens and collagen systems shows that each has advantages and disadvantages. The good initial development in the lens is partly due to the three-dimensional support it provides, while subsequent development probably fails because of the restriction of metabolic exchange by the dense lens material surrounding the embryo. Outgrowth on collagen, however, does not facilitate the maintenance of organization, as flattening of the embryo interferes with cellular interaction. The importance of this interaction is also suggested by the differentiation seen when ICMs fuse, producing a greater opportunity for cell-to-cell contact. Those embryos that do maintain their organization on collagen, however, have the advantage of being held at the medium/collagen interface, where metabolic exchange is less restricted, and in addition they escape deleterious interaction with the trophoblast which is drawn off over the collagen surface.

As far as maintenance of organization within the embryo is concerned, a system combining the support provided by the lens together with the more favourable exchange conditions on collagen would be ideal. Preliminary experiments using collagen gel into which embryos can be incorporated just before the gel sets, so that they can attach in a three-dimensional manner, suggest that this may be feasible.

\section{NUTRIENT FAGTORS IN EARLY DIFFERENTIATION AND IMPLANTATION}

Apart from physical factors affecting the organization of the embryo, differentiation in vitro will also be influenced by the provision of suitable nutrient requirements. As mentioned previously, embryos cultured in BSS plus albumin show neither trophoblast outgrowth nor any morphological signs of differentiation. In all the systems described above, in which some ICM differentiation was obtained, serum is a component of the medium, suggesting that it may supply a particular requirement for ICM differentiation which BSA alone does not.

\section{Behaviour of embryos on collagen in the absence of serum}

One of the requirements for the initiation of differentiation in vitro is trophoblast attachment. Without this, development does not occur, even in the presence of serum, as can be shown by culturing embryos on agar surfaces which do not facilitate cell attachment (Jenkinson, 1971). Outgrowth in vitro is normally dependent upon the presence of serum (Gwatkin, 1966a, b). This requirement can be fulfilled without serum, however, by the use of collagen surfaces. In this way it is possible to assess whether the function of serum in development in vitro is indirect, i.e. by allowing trophoblast outgrowth, or whether it also has a nutrient rôle.

Embryos cultured on collagen in BSS with BSA readily produce trophoblastic outgrowths. The cells of the ICM, however, never differentiate and remain, with little or no proliferation, as a group of rounded cells on the upper 
surface of the outgrowth. Even if several embryos are cultured together to allow ICM fusion, as described previously, so increasing the chance of cell-tocell interaction, development does not occur. Thus it would appear that while trophoblast attachment is important it is not the sole requirement for ICM development. The nutrient needs of the ICM are obviously different from those of the cleavage stages, which are met by relatively simple media.

Although ICM development is not found in these circumstances, the outgrowth of blastocysts on collagen substrates in serum-free media indicates that changes continue to occur in the trophoblast in the same simple media which supported its initial differentiation. These changes must involve alterations in the structure or composition of the surface of the trophoblast cells so as to lead to an increase in their adhesiveness for substrates possessing the surface characteristics of collagen. These changes occur only at the late-blastocyst stage since earlier stages placed on collagen after removal of the zona show no affinity for it, even if disaggregated, although the cells do show a great affinity for each other. The adhesive interactions involved in earlier development would appear to be qualitatively different from those involved in outgrowth.

It has been suggested that metabolic activation of the blastocyst, which can be brought about in vitro by the addition of serum, is necessary for outgrowth, indicating that blastocyst activation by uterine factors may be necessary for the initiation of implantation in vivo (Menke \& McLaren, 1970; McLaren \& Menke, 1971). However, the outgrowth of blastocysts in the absence of any serum activating factors, given a physically compatible surface, would indicate that there is no necessary correlation between activation and outgrowth and it suggests that the main rôle of serum in promoting outgrowth is to provide a suitable protein coating on glass surfaces.

An alternative mechanism for the control of implantation, based on cell surface changes, is suggested by the behaviour of the blastocyst on various physically different surfaces (Jenkinson \& Wilson, 1973). Outgrowth cannot take place until the late-blastocyst stage is reached, and, while the trophoblast can achieve the necessary differentiation in the simplest media, it cannot express it as outgrowth unless a physically suitable surface is provided. Implantation in vivo may similarly depend on the coincidence of this stage of blastocyst differentiation with the provision of a physically suitable surface on the otherwise refractory uterine epithelium. That hormonally controlled changes in the cell surface structure of the uterine epithelium do take place at the time of implantation is strongly suggested by the various ultrastructural studies of this stage.

\section{Development of embryos in lens fibre in the absence of serum}

Although serum appears to be a requirement for ICM development the nature of the rôle it fulfils is uncertain. Hsu (1972) has found that most commercially available FCS does not promote ectoderm and endoderm differentiation from the ICM. In those sera that do support differentiation the active component would appear to be a non-dialysable macromolecule, although it is uncertain whether this is a normal serum component, a protein/steroid complex or a compound similar to the uterine-specific glycoprotein produced near the 
time of implantation under hormonal influence. It has been suggested that such uterine-specific products may play an important part in controlling embryonic development (Krishnan \& Daniel, 1967).

We have found that mouse blastocysts cultured in lens protein in the absence of serum are capable of a degree of differentiation comparable to that which we have found in either lens or collagen cultures in the presence of serum. Because of the isolated position of the lens in the body and its total lack of vascularization, it is unlikely that it contains any uterine-specific product or any other circulating serum product produced under hormonal conditioning in the pregnant animal. In view of this it is more likely that the ability of the embryo to undergo differentiation into ectoderm and endoderm is intrinsic and depends neither upon direct hormonal influence nor upon a specific uterine product or steroid/protein complex with a gene-activator function. The ability of embryos to develop to some extent in ectopic sites, even in males, supports this conclusion.

On the basis of the development seen in lens cultures in the absence of serum we would suggest that the main requirement for the initiation of embryonic development is a ready supply of protein. To this extent the development of the embryo is dependent upon the mother since the total protein content of the uterine fluid is under maternal control. It would appear that, in the mouse, this protein content changes during the preimplantation stages, increasing as the time of implantation approaches (Gwatkin, 1969). In addition to the uterine fluid, a rich and diverse source of protein in the form of degenerating uterine cells, which are phagocytosed by the trophoblast, also becomes available at the time of implantation. Part of the value of lens material in supporting development in vitro may be due to the fact that the fibre particles can be phagocytosed by the trophoblast.

Whether any particular class of protein is especially valuable to the developing embryo is unknown. It might be envisaged that some proteins are more valuable than others, depending upon the spectrum of enzymes possessed by the embryo. The lens protein we have used is highly specific and well characterized, consisting of $\alpha, \beta$ and $\gamma$ crystallins. Since these proteins are easily separable it should be feasible to assess if any particular type is especially important for development in vitro. By combination of these lens proteins with the threedimensional collagen support system mentioned previously, it may prove possible to obtain not only initial development in a high percentage of cases but continued embryonic development, independently of variation in serum batches.

\section{IMMUNOLOGIGAL ASPECTS}

There is evidence that development in vitro from the eight-cell stage to the blastocyst can be inhibited by immune sera, indicating the presence of histocompatibility antigens on the cleavage-stage embryo (Heyner, Brinster \& Palm, 1969). However, the ability of blastocysts to undergo attachment and outgrowth is unaffected by the presence of immune lymphoid cells which are capable of completely destroying fibroblasts of the same genotype (Jenkinson $\&$ Billington, 1974). In addition, outgrowths of pure postimplantation trophoblast have been found to be unaffected even after pretreatment with neur- 
aminidase which is known to expose antigens masked by sialic acid (Billington, Elson, Jenkinson \& Searle, 1973). It would appear that during the course of development the antigenic expression on the trophoblast changes.

Preliminary results have also suggested that the ICM at the time of implantation is unaffected by immune lymphoid cells, whereas the postimplantation embryonic sac is susceptible to attack. With the further development of techniques for the support of implantation-stage embryos in vitro, it may be possible to analyse the differentiation of antigens throughout early development.

\section{ACKNOWLEDGMENT}

We are grateful to the A.R.C. for financial support.

\section{REFERENGES}

Barlow, P. W. \& Sherman, M. I. (1972) The biochemistry of differentiation of mouse trophoblast: studies on polyploidy. F. Embryol. exp. Morph. 27, 447.

Billington, W. D., Elson, J., Jenkinson, E. J. \& Searle, R. F. (1973) Antigenicity of the trophoblast. Proc. 1st Int. Congr. Immunalogy in Obstetrics and Gynaecology, Padua, 1973. Excerpta Medica (in press).

BRYson, D. L. (1964) Development of mouse eggs in diffusion chambers. Science, N.Y. 144, 1351.

COLE, R. J. \& PAUL, J. (1965) Properties of cultured pre-implantation mouse and rabbit embryos and cell strains derived from them. In Pre-Implantation Stages of Pregnancy, p. 82. Ed. G. E. W. Wolstenholme. Ciba Foundation Symposium. Churchill, London.

Elsdale, T. \& Bard, J. (1972) Collagen substrata for studies on cell behaviour. F. Cell Biol. 54, 626.

EhrmanN, R. L. \& Gey, G. O. (1956). The growth of cells on a transparent gel of reconstituted rat-tail collagen. F. natn. Cancer Inst. 16, 1375.

GARDNer, R. L. (1971) Manipulations on the blastocyst. Adv. Biosci. 6, 279. (Schering Symposium on Intrinsic and Extrinsic Factors in Early Mammalian Development. Pergamon, Vieweg.)

Gwatkin, R. B. L. (1966a) Defined media and development of mammalian eggs in vitro. Ann. N.Y. Acad. Sci. 139, 79.

Gwatkin, R. B. L. (1966b) Amino acid requirements for attachment and outgrowth of mouse blastocysts in vitro. 7. cell. Physiol. 68, 335.

Gwatkin, R. B. L. (1969) Nutritional requirements for post-blastocyst development in the mouse. Int. F. Fert. 14, 101.

Heyner, S., Brinster, R. L. \& Palm, J. (1969) Effect of iso-antibody on pre-implantation mouse embryos. Nature, Lond. 222, 783.

Hsu, Y. G. (1971) Post-blastocyst differentiation in vitro. Nature, Lond. 231, 100.

Hsu, Y. G. (1972) Differentiation in vitro of mouse embryos beyond the implantation stage. Nature, Lond. 239, 200.

Jenkinson, E. J. (1971) In vitro development and implantation of the mouse embryo. Ph.D. thesis, University of Wales.

Jenkinson, E. J. \& Billington, W. D. (1974) Differential susceptibility of mouse trophoblast and embryonic tissue to immune cell lysis. Transplantation (in press).

Jenkinson, E. J. \& Wilson, I. B. (1970) In vitro support system for the study of blastocyst differentiation in the mouse. Nature, Lond. 228, 776.

Jenkinson, E. J. \& Wilson, I. B. (1973) In vitro studies on the control of trophoblast outgrowth in the mouse. F. Embryol. exp. Morph. 30, 21.

KRishnan, R. S. \& Daniel, J. G. (1967) 'Blastokinin' inducer and regulator of blastocyst development in the rabbit uterus. Science, N.X. 158, 490.

McLaren, A. \& MENKE, T. M. (1971) $\mathrm{CO}_{2}$ output of mouse blastocysts in vitro, in normal pregnancy and in delay. 7. Reprod. Fert., Suppl. 14, 23.

Menke, T. M. \& MaLaren, A. (1970) Mouse blastocysts grown in vivo and in vitro: carbon dioxide production and trophoblast outgrowth. F. Reprod. Fert. 23, 117.

MinTz, B. (1964) Formation of genotypically mosaic mouse embryos and early development of lethal $\left(\mathrm{t}^{12} / \mathrm{t}^{12}\right)$ normal mosaics. J. exp. Zool. $157,85$. 